\title{
Coreflood Study of Effect of Surfactant Concentration on Foam Generation in Porous Media
}

\author{
G. Yu* and W.R. Rossen* \\ Delft University of Technology, Delft, 2628CN / 2600GA, Netherlands
}

\section{S. Vincent-Bonnieu*}

Shell Global Solutions International B. V., Amsterdam, Netherlands

\section{Supporting Information}

\begin{abstract}
The propagation of foam in an oil reservoir depends on the creation and stability of the foam in the reservoir, specifically the creation and stability of foam films, or lamellae. As the foam propagates far from the injection well, superficial velocity and pressure gradient decrease with distance from the well. Experimental (Friedmann et al. Steam-foam mechanistic field trial in the midway-sunset field. SPERE. 1994, 9 (4), 297-304) and theoretical (Ashoori, et al. Roles of Transient and Local Equilibrium Foam Behaviour in Porous Media: Traveling Wave. Colloids Surf. A 2011, 337 (1-3), 228-242). studies relate concerns about foam propagation at low superficial velocity to the minimum velocity or pressure gradient for foam generation near the well (Gauglitz et al. Foam Generation in Homogeneous Porous Media. Chem. Eng. Sci. 2002, 57, 4037-4052; Rossen et al. Percolation Theory of Creation and Mobilization of Foams in Porous Media. AI Chem Eng. J. 1990, 36, (8)). The objective of this work is to measure the impact of surfactant concentration and gas fractional flow on foam generation. Theory (Kam et al. Model for Foam Generation in Homogeneous Media. SPE J. 2003, 8 (4): 417-42, SPE-87334-PA; Rossen 1990) relates foam generation to gas fractional flow and, indirectly, to the stability of foam films, or lamellae, which in turn depends on surfactant concentration (Apaydin et al. Surfactant Concentration and End Effects on Foam Flow in Porous Media. (Apaydin et al.Transp Porous Media. 2001, 43, 511-536). However, the link between foam generation and surfactant

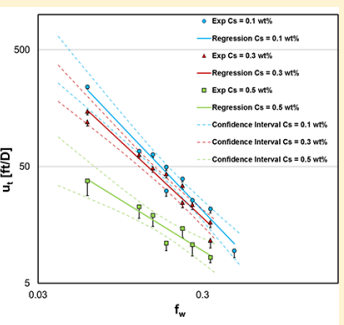
concentration has not been established experimentally. In our experiments, nitrogen foam is generated in a core of Bentheimer sandstone. The foam-generation experiments consist of measuring the minimum velocity for foam generation as a function of gas fractional flow at three surfactant concentrations well above the critical micelle concentration. Experimental results show that the minimum velocity for foam generation decreases with increasing liquid fraction, as shown by previous foam generation studies (Friedmann et al., 1994; Rossen and Gauglitz, 1990). Additionally, our results show that this velocity decreases with increasing surfactant concentration, far above the CMC. We also propose a workflow for screening out the experimental artifacts that can distort the trigger velocity.
\end{abstract}

\section{INTRODUCTION}

Gas-injection enhanced oil recovery (EOR) can efficiently displace oil. ${ }^{19,21,26}$ However, gas-injection EOR suffers from poor sweep efficiency and may achieve limited oil recoveries in field applications, ${ }^{19,26}$ primarily due to low gas viscosity (leading to fingering and channelling), low gas density (leading to gravity override) and geological heterogeneity. Reducing the relative mobility of gas thus becomes a major challenge for gasinjection EOR. Foam can provide mobility control for gas flooding. Foam is a dispersion of gas bubbles in an aqueous phase, stabilized by surfactant molecules at the gas-liquid interfaces. When foam is generated in porous media, the flow paths of gas are blocked by liquid films, or lamellae, while the liquid phase remains continuous. The lamellae blocking the gas phase add additional capillary resistance to gas flow and thereby make the gas phase less mobile.
The conditions for foam generation depend in part on the method of injection. In our experiments, we consider steady gas and liquid injection at a fixed gas fraction, where gas has already been injected for a time before surfactant is added to the system. ${ }^{25}$ This initial state is relevant to the propagation of a foam front far from a well, where alternating slugs of gas and liquid have mixed and where gas has advanced ahead of the foam front. During these steady-state experiments, foam is created in the porous medium by coinjecting gas and surfactant solution at a fixed gas fraction; foam generation requires exceeding a minimum superficial velocity $u_{\mathrm{t}}^{\mathrm{min}}$, or pressure gradient $\nabla p^{\min }{ }^{25}$ It is pressure gradient $\nabla p$, not total

Received: August 13, 2018

Revised: October 11, 2018

Accepted: November 30, 2018

Published: November 30, 2018 

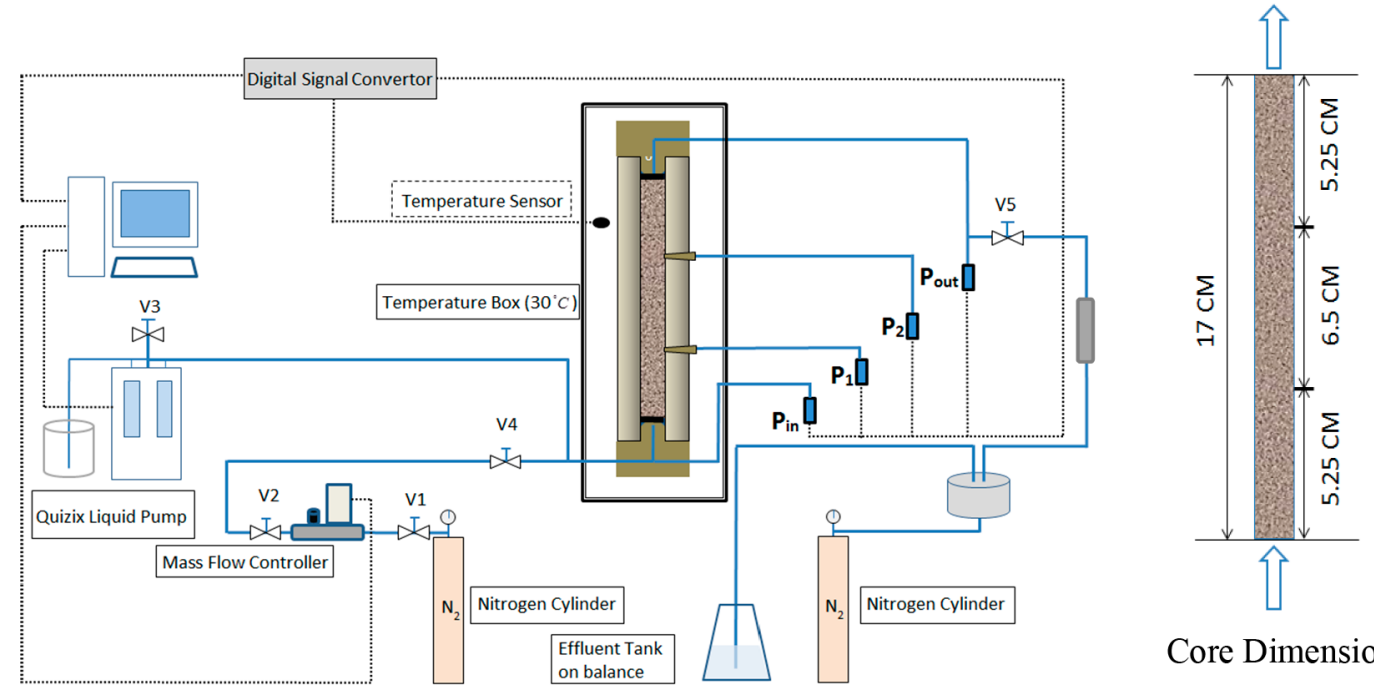

Core Dimension

Figure 1. Experimental apparatus for foam-generation experiments. The core is mounted vertically in an oven at a temperature of $30{ }^{\circ} \mathrm{C}$. Four absolute-pressure meters are connected along the core, with pressure ranges of 120 bar. Gas and liquid are injected from the bottom and exit from the top. A small metal container is connected between the last pressure meter $P_{\text {out }}$ and the back-pressure regulator to stabilize pressure in the outlet section of the core.

superficial velocity $u_{v}$ that triggers foam generation, but results are often reported in terms of $u_{\mathrm{t}}^{\text {min }}$, which is easier to control and measure in the laboratory. "Foam generation", in this context, refers to an abrupt jump from a state of high gas mobility to one of very low mobility. This abrupt change depends on the rate of lamella creation exceeding the rate of lamella destruction in the porespace, ${ }^{6,16}$ leading to a spontaneous run-away process and a jump in state. ${ }^{13,15}$ In this paper, we refer to this minimum pressure gradient or superficial velocity as the "trigger" for foam generation.

The triggers $u_{\mathrm{t}}^{\min }$ or $\nabla p^{\mathrm{min}}$ depend on gas fractional flow (foam quality $f_{\mathrm{g}}$ ). Greater $f_{\mathrm{g}}$ requires a greater velocity to trigger foam generation. ${ }^{25}$ In the vicinity of an injection well, in situ foam generation and foam propagation are usually easy due to large superficial velocity and pressure gradient. The real concern for generation and propagation, therefore, lies in locations far from the injection well, where both superficial velocity and pressure gradient are low. ${ }^{1,8}$ Hence, the minimum velocity for foam generation and propagation in porous media is of great importance to foam application.

Previous experimental studies have not identified a connection between the minimum velocity for foam generation and surfactant concentration. The mechanisms of individual lamella generation (leave-behind, snap-off, lamella mobilization) are not believed to depend on the presence of surfactant. ${ }^{9,24}$ For a given homogeneous porous medium, the trigger velocity or pressure gradient for foam generation depends on the capillary resistance of a lamella to be displaced from a pore throat and subsequent division. ${ }^{25}$ This resistance is of course proportional to the gas-liquid surface tension $\gamma$. Therefore, the minimum condition for foam generation depends on surface tension, but this dependence affects foam generation only for surfactant concentrations below the CMC.

The survival of lamellae once created, however, does depend on surfactant formulation and concentration. ${ }^{26}$ Foam generation therefore requires not only production of lamellae in the porous medium, but also the survival of the newly created lamellae. The greater the lamella-destruction rate (either due to ineffective surfactant or insufficient surfactant concen- tration), the greater the lamella-creation rate needed to generate foam. The stability of foam in porous media, reflected in the limiting capillary pressure $P_{\mathrm{c}}$ * or water saturation $S_{\mathrm{w}}{ }^{*}$ for foam stability, increases with increasing surfactant concentration far above the Critical Micelle Concentration (CMC). ${ }^{2,12}$ Therefore, one would expect that increasing surfactant concentration reduces the minimum superficial velocity or pressure gradient for foam generation by reducing the rate of lamella breakage. However, this link has not been demonstrated experimentally. In this paper we present experimental verification of the connection between the minimum velocity for foam generation and surfactant concentration for one surfactant formulation. We also propose a workflow for identifying the triggering velocity and screening out the experimental artifacts. We relate the experimental results to a population-balance model for foam generation. The model agrees with the trends of the experimental results.

\section{EXPERIMENTS ON FOAM GENERATION}

Experimental Method and Materials. In our experiments, foam is generated in situ by coinjecting surfactant solution and nitrogen into a homogeneous Bentheimer sandstone core at a back-pressure of $40 \mathrm{bar}$ and a temperature of $30^{\circ} \mathrm{C}$. The main objective of our experiments is to map out the minimum total superficial velocity $u_{\mathrm{t}}^{\min }$ required to trigger foam generation for different foam qualities (gas fractional flow) $f_{\mathrm{g}}$ and three surfactant concentrations $C_{\mathrm{s}}$, each far above the critical micelle concentration, CMC. Based on the measurement of the CMC by Jones et al., ${ }^{12}$ all three surfactant concentrations are far above the $\mathrm{CMC}$, which is approximately 0.005 wt $\%$ for AOS with 3.0 wt $\% \mathrm{NaCl}$.

We use the same surfactant, Sodium C14-16 Alpha Olefin Sulfonate (AOS-1, Bioterge AS-40), for all experiments. Both brine and surfactant solutions contain $3 \mathrm{wt} \% \mathrm{NaCl}$. Figure 1 shows the experimental apparatus. The Bentheimer core is 17 $\mathrm{cm}$ in length, with a diameter of $1 \mathrm{~cm}$. The permeability of the core is $1.87 \times 10^{-12} \mathrm{~m}^{2}$. Four absolute-pressure transducers are located along the core. Two of them are located on the inlet and outlet lines, respectively, whereas the other two are in 


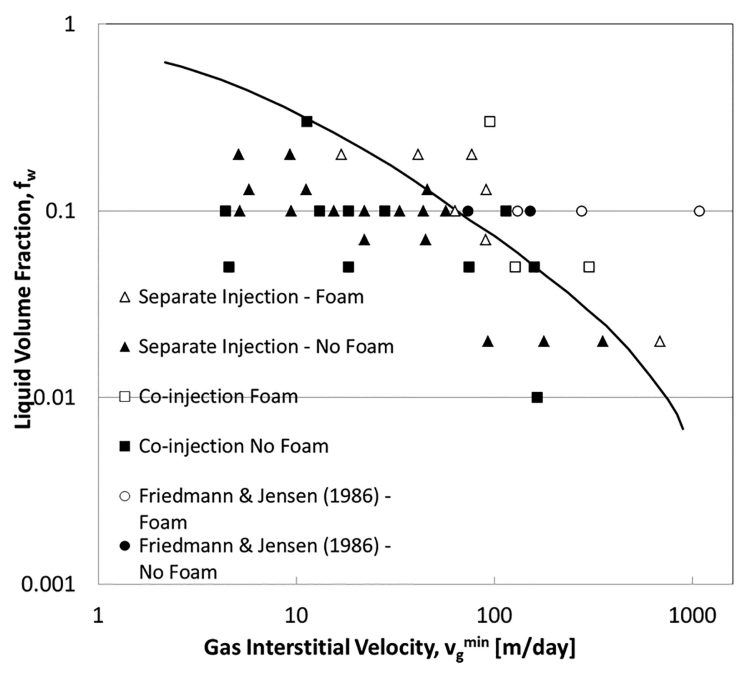

(a)

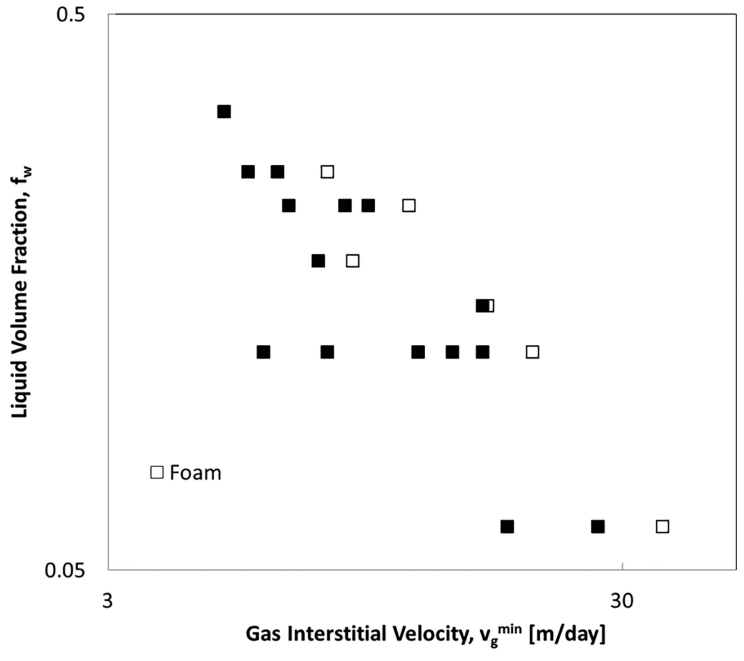

(b)

Figure 2. (a) Minimum gas interstitial velocity required to trigger foam generation as a function of injected liquid volume fraction (or $f_{\mathrm{w}}$, i.e., $(1-$ $\left.f_{\mathrm{g}}\right)$ ). The plot is reproduced from data of Rossen and Gauglitz. ${ }^{25}$ Trends superimposed on data are from a percolation-theory analysis for foam generation described in Rossen and Gauglitz. ${ }^{25}$ (b) A similar plot based on data from our experiments $\left(C_{s}=0.5\right.$ wt $\left.\%\right)$. White dots represent the observed trigger velocity for the given injected liquid volume fraction, and black dots represent the velocities tested before the trigger of foam generation.

direct contact with the core. The core is thus divided into three sections, with inlet and outlet sections $5.25 \mathrm{~cm}$ long, and the middle section $6.5 \mathrm{~cm}$ long (Figure 1). Three different surfactant concentrations are tested for impact on foam generation: $0.1,0.3$, and $0.5 \mathrm{wt} \%$ (Supporting Information (SI) Table S1). Surface tensions of the three surfactant solutions are shown in SI Table S2.

A small pressure cell of volume $150 \mathrm{~mL}$ lies between the core and the back-pressure regulator (BPR) to mitigate any fluctuations at the BPR. Since, as mentioned above, pressure gradient is thought to play an essential role in foam generation, any sudden increase or decrease in back-pressure would lead to an abrupt change in pressure gradient at the outlet of the core. In such cases, foam generation could be triggered near the outlet.

The core is initially fully saturated with brine. Then $\mathrm{N}_{2}$ and brine are coinjected at constant gas fractional flow. After steady state is achieved, brine injection is replaced by injection of surfactant solution at the same injection rate and fractional flow of gas. After 1 pore volume of surfactant solution has been injected, we begin the process of raising superficial velocity in steps until foam generation is triggered. At each step, we wait for a time to see if foam generation has occurred; details are given below. The trigger for foam generation could lie between the measured velocity at which foam generation occurs and the velocity just before it. The resulting uncertainty range for each experiment is illustrated by the error bars in the results shown below.

Experimental Artifacts and Screening Criteria. Our goal is to determine the velocity at which foam generation occurs in steady flow in a homogeneous porous medium. Identification of the foam trigger (with regard to either velocity or pressure gradient) can be problematic, and experimental results are typically scattered, as illustrated in Figure 2. There are at least two experimental artifacts that contribute to the scatter: (1) the "incubation effect", and (2) the capillary end effect. Both effects may lead to foam generation at superficial velocities lower than the minimum velocity $u_{t}^{\min }$. These two effects are described below.

Baghdikian and Handy, ${ }^{3}$ injecting liquid and gas into cores at steady, low velocities, observed a slow increase in $\nabla p$ until, many hours or even days later, there was an abrupt increase in $\nabla p$ over a period of minutes or hours: that is, "foam generation". They call this foam generation occurring after a delay the "incubation effect" (see refs 4, 11, and 26). The reason for this behavior is not clear, but it is likely the result of an accumulation of local perturbations in flow rates, foam quality, and capillary pressure, etc. over time, leading to creation of static lamellae and increasing pressure gradient. ${ }^{26}$ We exclude these cases from our results, because we want to identify the point where velocity or pressure gradient triggers foam generation without the effects of extraneous fluctuations accumulated over time.

The capillary end effect ${ }^{5,18,23}$ is another complicating artifact in foam-generation experiments. Apaydin and Kovscek ${ }^{2}$ studied the role of surfactant concentration and end effects on foam flow in porous media. The classic capillary end effect is an accumulation of water near the outlet face of the porous medium caused by contact with fluid outside the porous medium at a capillary pressure of zero or near zero. The wet conditions near the core outlet are ideal for foam generation. ${ }^{24}$ At larger surfactant concentrations, Apaydin and Kovscek ${ }^{2}$ reported, the end effect results in a larger pressure gradient building first near the outlet and propagating upstream, against the direction of flow, toward the inlet. Similar effects, where a large increase in pressure gradient first occurs near the outlet and then propagates upstream. Similar results are reported by Nguyen et al. $^{22}$ and Simjoo et al. $^{27}$ The mechanism of upstream propagation of a stronger foam state is unclear, but, in any case, the origin of the state is a result of the capillary end effect, and therefore it is not representative of a homogeneous porous medium. Hence, we exclude cases in which a large pressure gradient is created near the outlet and then propagates to or disturbs upstream core sections. 
We define the trigger as the total superficial velocity at which foam is created quickly near the core inlet, without a long period of steady injection or propagation of foam first created near the outlet. Below we define the criteria to define a valid trigger velocity and to identify unacceptable cases. Figure 3

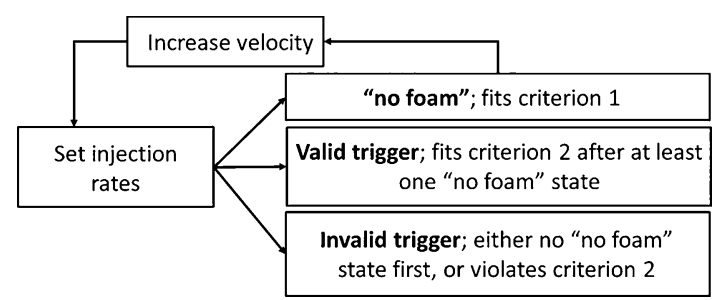

Figure 3. Experimental procedures for identification of a valid trigger velocity. Each experiment should begin at a superficial velocity lower than the trigger velocity. Three possible scenarios could happen at a particular velocity. (1) If no foam is created at this velocity (criterion $1)$, then a stepwise increase of superficial velocity is required, until a valid trigger, at which foam generation begins, is identified. (2) If foam generation takes place (meeting all conditions specified in criterion 2) after at least one "no foam" state, then a valid trigger velocity is identified. (3) If foam generation takes place at the very first injection rate, or any event(s) that violate criterion 2 take place during the process of velocity increase, the experiment is be aborted and repeated, until it meets both criteria and a valid trigger is identified.

illustrates how we identify a valid trigger according to two criteria:

1. The experiment should begin with at least one velocity lower than the trigger velocity for foam generation. In Figure 3 we call this state "no foam" for simplicity. In reality, it could be a state with a modest reduction of gas mobility, or what Ransohoff and Radke ${ }^{24}$ refer to as a "leave-behind foam." At this velocity, there should be no significant pressure drop in any core section. There are two criteria to define the condition before the trigger:

a. Pressure gradient along the entire core increases within the next 10-20 s upon the increase of superficial velocity, and settles down to a new steady state quickly (usually within 20-30 s). When the new steady state is achieved, the increase in pressure drop is of the same magnitude as the proportional increase in velocity from the previous step. Ideally this rule applies to all core sections. In many cases, however, the $\Delta P$ across the outlet section increases much more than proportionately with the velocity increase, and more than the pressure drop in other sections. We accept cases with a modest $\Delta P$ in the outlet section (no more than $1 \mathrm{bar}$, too little to affect gas volume or superficial velocity upstream) if the state of large $\nabla p$ does not migrate upstream to the second section. In other words, if there is foam generation near the outlet but this is not the cause of subsequent foam generation near the inlet, we accept that case.

b. Pressure gradient along the core should remain constant, without an upward trend, once a steady state is achieved. The period during which a steady pressure gradient is verified should be limited to avoid the "incubation effect" (see criterion 2a, below). We checked the steadystate of an injection rate for about $15-20 \mathrm{~min}$, before raising injection rate to the next level. If the injection period lasts for more than $40-60 \mathrm{~min}$, the incubation effect could compromise the validity of result.

2. The trigger should be characterized by a rapid increase in pressure drop in all sections while keeping injection rate and foam quality constant. Specifically

a. The pressure drop across the first section rises steeply in the first section within $2-5 \mathrm{~min}$ of the increase in injection rate. The zone of large pressure gradient propagates from the first section downstream, but not from the last section upstream. A pressure rise occurring after, say, an hour of injection at a given rate could be a symptom of the incubation effect and unreliable.

b. At the trigger, the magnitude of increase in $\Delta P$ is larger, and the period to reach the new steady state is longer $(20-40 \mathrm{~min})$, than in the steps before the trigger. The magnitude of gradient of the newly formed steady-state should be substantially greater (10-10 times) than the pressure gradient before the trigger.

If and only if both criteria are satisfied in our experiment, we identify the minimum velocity for generation for the given surfactant concentration and foam quality. We denote this total superficial velocity as $u_{\mathrm{t}}^{\min }$ below. If any of the above criteria are violated, the result of this experiment is discarded. The experiment should be repeated until a valid trigger is identified.Figure 4 shows examples of both valid (Figure $4 a$ ) and invalid (Figure 4b) experimental results.

\section{RESULTS}

Our results (Figures 5 and 6) show that (1) the minimum superficial velocity $u_{\mathrm{t}}^{\min }$ required to trigger foam generation increases with decreasing liquid fractional flow $f_{\mathrm{w}}$, and (2) $u_{\mathrm{t}}{ }^{\text {min }}$ decreases with increasing surfactant concentration in the aqueous phase. Foam generation becomes easier for wetter foam (greater $f_{\mathrm{w}}$ ) and higher surfactant concentration, even far above the CMC. The trend on this $\log -\log$ plot (Figure 5) is roughly linear for each surfactant concentration. There is some scatter in the data, as in Figure 2, and some overlap between the data at some surfactant concentrations.

Figure 6 shows the regression lines as well as the 95\% confidence intervals for the trends ${ }^{28}$ for the three surfactant concentrations used in our experiments. Although there is some overlap between the data for different surfactant concentrations, there is relatively little overlap between the confidence intervals for the trends at 0.1 and $0.3 \mathrm{wt} \%$ concentrations. There is no overlap between the top two trends and that at the bottom for 0.5 wt \% concentration. In summary, surfactant concentration has an effect on foam generation that transcends the scatter in the individual data.

\section{MODELING THE FOAM TRIGGER}

The population-balance model of Kam and Rossen ${ }^{13}$ and its variants $^{14,15}$ is the only population-balance model that explains the minimum velocity for foam generation seen in experiments. ${ }^{10}$ Like other population-balance models, this model represents foam texture explicitly, with rates of lamella creation and lamella coalescence defined by two functions. In this 

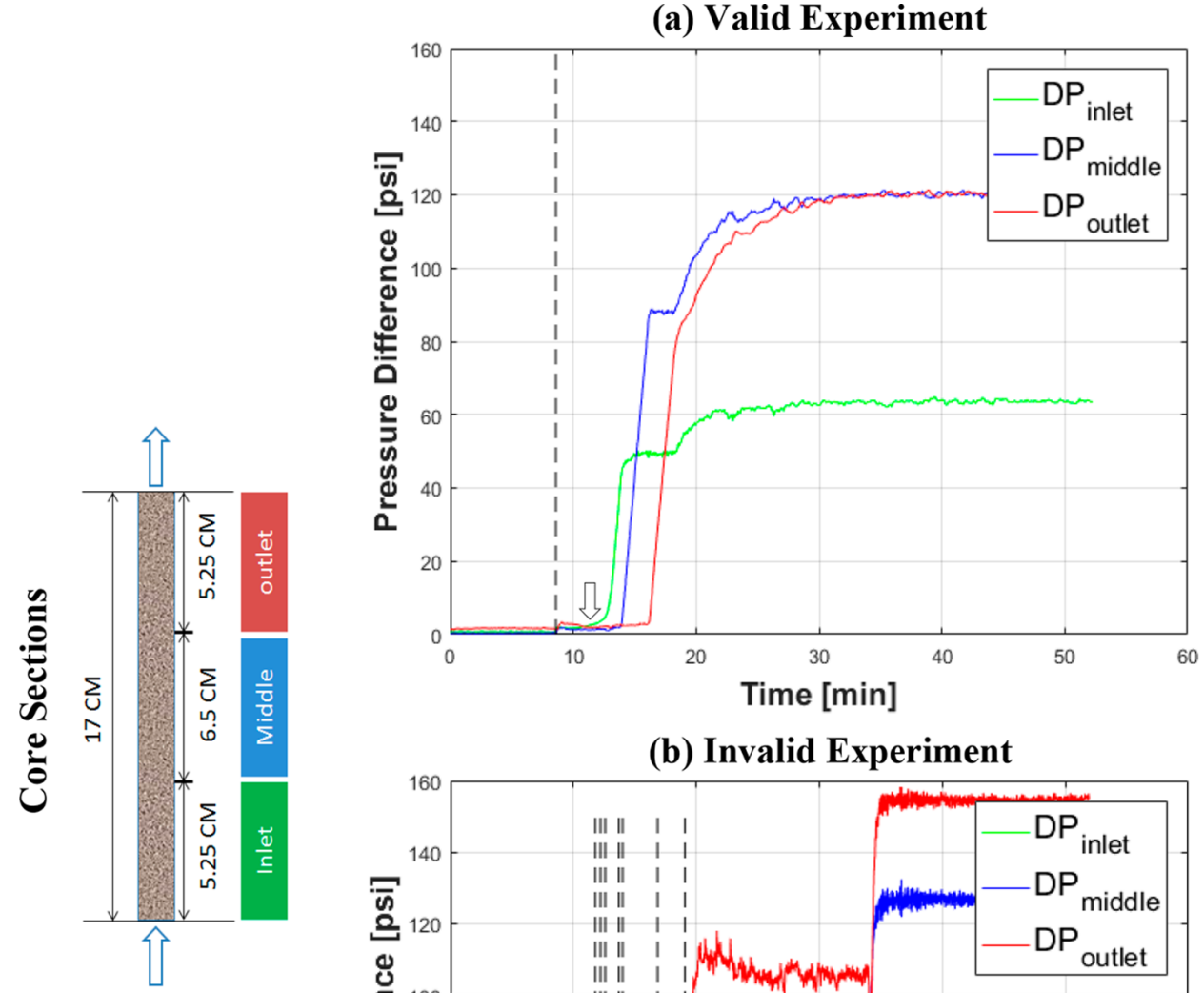

(b) Invalid Experiment

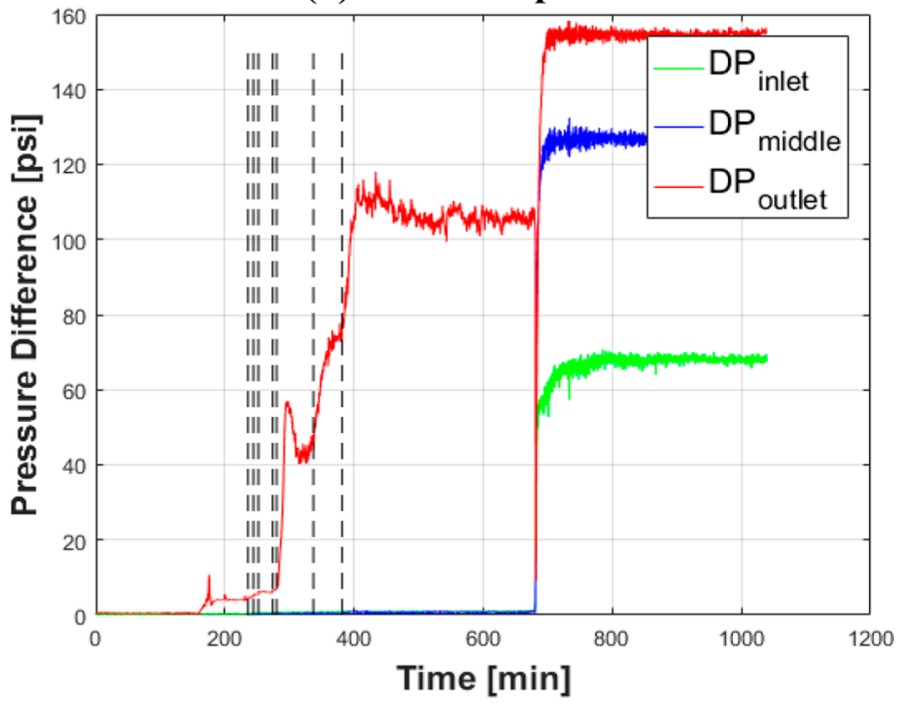

Figure 4. (a) A valid finding of a trigger velocity $\left(C_{\mathrm{s}}=0.3 \mathrm{wt} \%, f_{\mathrm{g}}=85.04 \%\right)$. Upon the increase in injection rate at after about $81 / 2$ min coinjection of surfactant solution and nitrogen, foam generation is triggered in the inlet section within 5 min and propagates downstream. (b) An invalid result $\left(C_{\mathrm{s}}=0.3 \mathrm{wt} \%, f_{\mathrm{g}}=87.98 \%\right)$. Weak foam is first created in the outlet section (at around $160 \mathrm{~min}$.) instead of upstream sections, likely due to end effect. Strong foam is created later near the outlet after a long period of injection (around $7 \mathrm{~h}$ ), and eventually pressure drop in the last section $(110 \mathrm{psi})$ is large enough to affect superficial velocities upstream. Foam finally fills the core after about $700 \mathrm{~min}(12 \mathrm{~h})$, but the effect of the last section cannot be ruled out.

model, the rate of lamella creation depends on pressure gradient. Similar to other population-balance models, the rate of lamella destruction is controlled by water saturation and the limiting water saturation $S_{\mathrm{w}}^{*}$, a parameter related to the limiting capillary pressure for foam destruction, $P_{c}^{*}$ via the capillary-pressure/saturation function $P_{c}\left(S_{w}\right) \cdot{ }^{2,17,20,29}$ As noted above, the process of lamella creation is not believed to depend on surfactant concentration; this assumption is incorporated into various population-balance models. ${ }^{7,13,16} S_{\mathrm{w}}{ }^{*}$ and $P_{\mathrm{c}}{ }^{*}$ do depend on surfactant concentration far above the CMC.,12

Figure 7 shows the relationship between pressure gradient and superficial velocity predicted by the model for one value of $S_{\mathrm{w}}{ }^{*}$. The trigger for foam generation is the maximum velocity on the lower (weak-foam) branch, where the function bends back toward lower values of superficial velocity. The values of $f_{\mathrm{w}}$ and $u_{\mathrm{t}}$ at this maximum represent the relation between foam quality and minimum velocity for foam generation for one value of $S_{\mathrm{w}}{ }^{*}$. Figure 8 shows how the trend shifts with $S_{\mathrm{w}}{ }^{*}$ and, by implication, with surfactant concentration.

The trend in superficial velocity $u_{t}$ against pressure gradient $\nabla p$ predicted by the model of Kam and Rossen ${ }^{13}$ (Figure 8) is similar to the experimental results in Figures 5 and 6. The model parameters (SI eqs A1 and A2, Table S2) were fit to data for a different foam formulation in a different porous medium. We present the model results with this set of parameters merely to indicate the trend predicted by the model. A quantitative fit would require fitting all the parameters, possibly tweaking the functional forms used to represent lamella creation as a function of $\nabla P$ and lamella destruction as a function of $S_{\mathrm{w}}$ in the model, and determining the relation between $S_{\mathrm{w}}{ }^{*}$ and surfactant concentration for this surfactant formulation in our porous medium. 


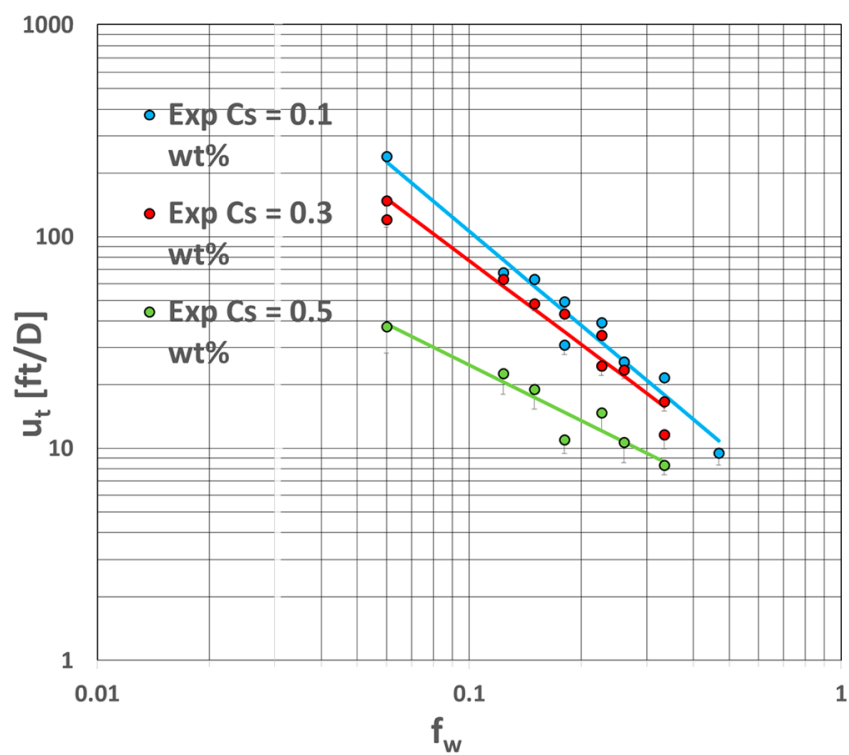

Figure 5. Experimental results for the trigger velocity for foam generation versus liquid fractional flow $f_{\mathrm{w}}$ for three different surfactant concentrations. Data plotted on log-log scale approximate a linear trend (solid lines) for each surfactant concentration; the least-squares fit to each trend is also shown. The error bars (below data points) represent the difference between the trigger velocity and the velocity tested immediately before it.

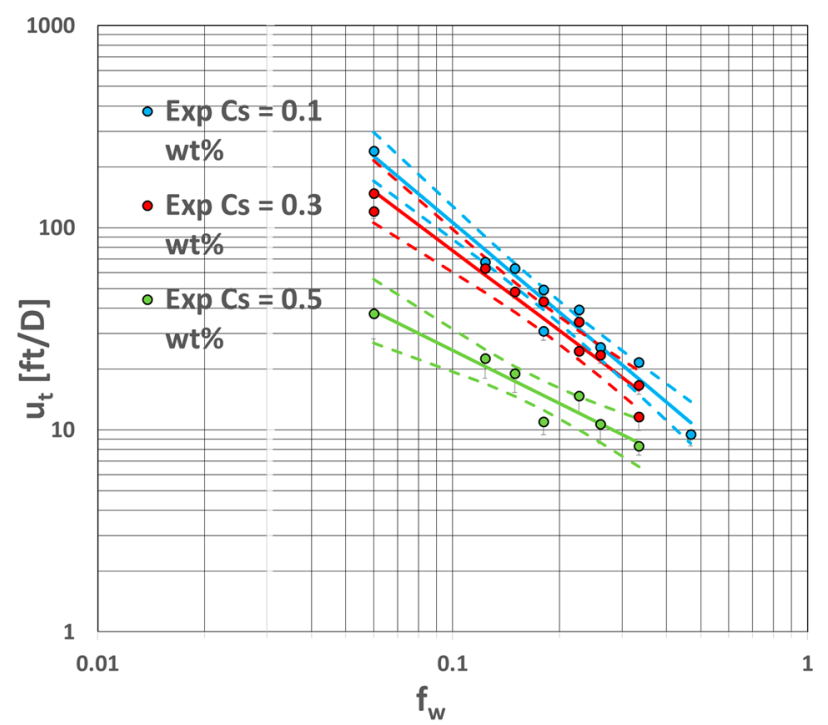

Figure 6. Estimated linear regression lines (solid lines) and 95\% confidence intervals (dashed curves) for the underlying trends of the three surfactant concentrations. Markers represent the experimental results, as in Figure 5.

\section{CONCLUSIONS}

1. Our data show that the minimum velocity for foam generation in steady flow decreases with increasing surfactant concentration and increasing injected liquid fractional flow $\left(f_{\mathrm{w}}\right)$.

2. The impact of surfactant concentration on foam generation that we find in our results is in accord with the prediction of Kam and Rossen's population-balance model, ${ }^{4}$ where the trigger velocity for foam generation increases with increasing foam quality $f_{\mathrm{g}}$, and decreases

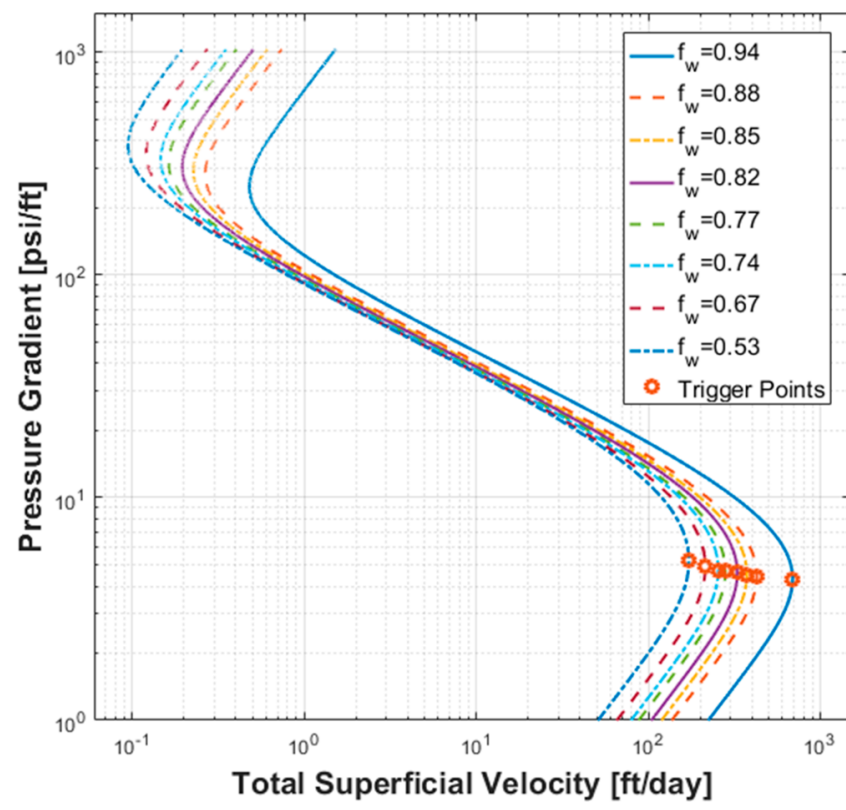

Figure 7. Steady-state total superficial velocity $u_{t}$ as a function of pressure gradient $\nabla \mathrm{p}$ for given foam qualities $f_{\mathrm{g}}$, from the populationbalance model of Kam and Rossen ${ }^{13}$ with parameters from SI (specifically, $S_{\mathrm{w}}{ }^{*}=0.201, S_{\mathrm{wc}}=0.2$ ). The lower branch represents the steady state of weak foam (or no foam); the upper branch represents the steady state of strong foam. The trigger for foam generation is the maximum of the lower branch (orange circles), where the $\nabla p\left(u_{\mathrm{t}}\right)$ function bends back to lower superficial velocities. These maximum values produce the blue curve in Figure 8. In an experiment at fixed superficial velocity, there would be a jump from the weak/no-foam state to the strong-foam state at the maximum of the lower branch.

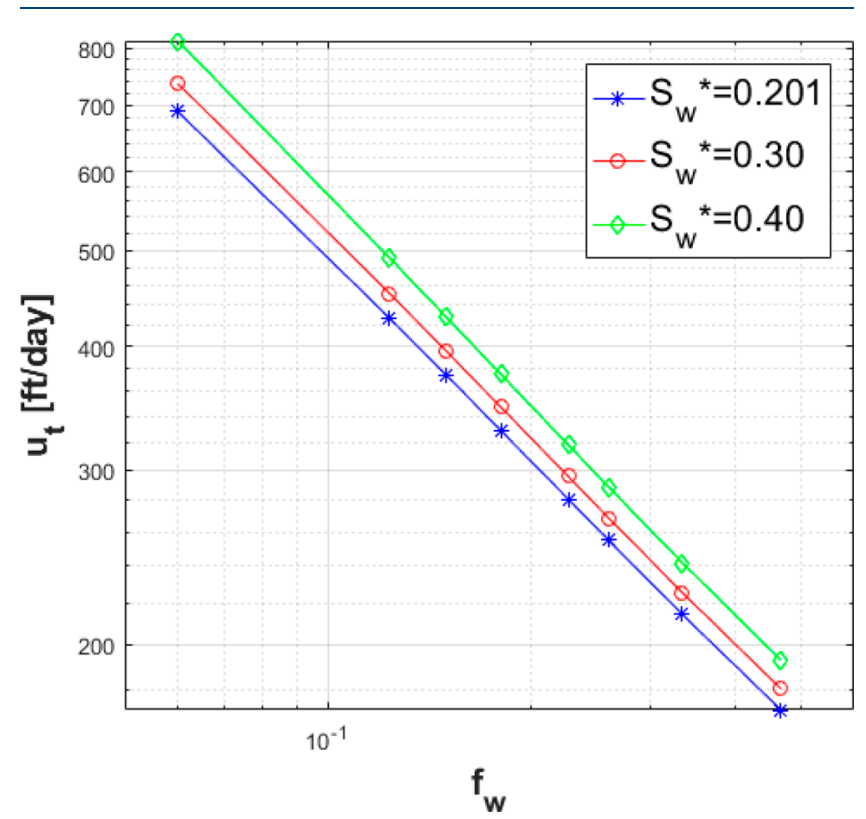

Figure 8. Model prediction of minimum superficial velocity for foam generation as a function of liquid fractional flow $f_{\mathrm{w}}$ and limiting liquid saturation $S_{\mathrm{w}}{ }^{*}$.

with increasing surfactant concentration $C_{\mathrm{s}}$ (reflected as $S_{\mathrm{w}}{ }^{*}$ in Kam and Rossen's model). This reflects an indirect link between lamella stability and "foam generation," because creation of foam in porous media depends on the stability of lamellae. 
3. Foam generation is closely related to foam propagation. The stability and transport of bubble transport at the leading edge of displacement front requires further investigation. However, our results suggest that foam propagation has a similar dependency on water fractional flow and surfactant concentration: wetter foam and greater surfactant concentration promote the transport of foam, even at surfactant concentrations far above the CMC.

\section{ASSOCIATED CONTENT}

\section{S Supporting Information}

The Supporting Information is available free of charge on the ACS Publications website at DOI: 10.1021/acs.iecr.8b03141.

(1) Table of foam qualities and surfactant concentrations in our experiment. (2) Input functions and coefficients for Kam and Rossen's population-balance model. $^{13}$ (3) Examples of pressure difference profile in our foam generation experiment. (PDF)

\section{AUTHOR INFORMATION}

\section{Corresponding Authors}

*(G.Y.) E-mail: G.Yu@tudelft.nl.

*(W.R.R.) E-mail: W.R.Rossen@tudelft.nl.

*(S.V.-B.) E-mail: S.VincentBonnieu@shell.com.

\section{ORCID}

G. Yu: 0000-0001-6133-8468

Notes

The authors declare no competing financial interest.

\section{ACKNOWLEDGMENTS}

This project is part of the Joint Industry Project (JIP) on foam for EOR. We thank and acknowledge both the sponsorship and advice of sponsoring companies and their representatives. S.V.B. thanks Sell Global Solutions International B.V. especially for their review on this article and their permission for publication. In addition, we thank Michiel Slob and Jolanda van Haagen Donker for their technical assistance with our experiments.

\section{NOMENCLATURE}

$C_{\mathrm{g}}=$ model parameter (SI Table S2)

$C_{\mathrm{c}}=$ model parameter (SI Table S2)

$C_{\mathrm{s}}=$ surfactant concentration, expressed as [wt \%]

$f_{\mathrm{g}}=$ gas fractional flow

$f_{\mathrm{w}}=$ water fractional flow

$k=$ permeability, $\left[\mathrm{m}^{2}\right]$

$k_{\mathrm{rg}}=$ gas relative permeability in absence of foam

$k_{\mathrm{rw}}=$ water relative permeability

$m=$ model parameter (SI Table S2)

$n=$ model parameter (SI Table S2)

$n_{\mathrm{f}}=$ foam texture or density, inversely related to bubble size (eq A.2), $\left[\mathrm{m}^{-3}\right]$

$\Delta P=$ magnitude of pressure gradient

$\Delta P=$ pressure drop across core or section of core

$\nabla P^{\min }=$ minimum pressure gradient required to trigger foam generation

$P_{C}=$ capillary pressure $[\mathrm{Pa}]$

$P^{*}{ }_{C}=$ limiting capillary pressure $[\mathrm{Pa}]$

$S{ }^{*}{ }_{w}=$ limiting water saturation-water saturation at limiting capillary pressure

$S_{\mathrm{gr}}=$ trapped/residual gas saturation
$S_{\mathrm{w}}=$ water saturation

$S_{\mathrm{wc}}=$ connate water saturation (eq $\mathrm{S} 1$ )

$u_{\mathrm{g}}=$ gas superficial velocity (Darcy velocity), $[\mathrm{m} / \mathrm{s}]$ in calculations, $[\mathrm{ft} / \mathrm{D}]$ in figures and texts

$u_{\mathrm{w}}=$ water superficial velocity (Darcy velocity), $[\mathrm{m} / \mathrm{s}]$ in calculations, $[\mathrm{ft} / D]$ in figures and texts

$u_{\mathrm{t}}=$ total superficial velocity (Darcy velocity), $[\mathrm{m} / \mathrm{s}]$ in calculations, $[\mathrm{ft} / D]$ in figures and texts

$u_{\mathrm{t}, \mathrm{c}}=$ minimum total superficial velocity (Darcy velocity) required for triggering of foam generation, $[\mathrm{m} / \mathrm{s}]$ in calculations, $[\mathrm{ft} / D]$ in figures and texts

$v_{\mathrm{g}}^{\min }=$ minimum gas interstitial velocity required for triggering of foam generation, defined in Figure 2

$\mu_{g}^{0}=$ gas viscosity in absence of foam [Pa s]

$\mu^{0} w=$ water viscosity [Pa s]

$\varphi=$ porosity

$\gamma=$ surface tension (SI Table S2), shown here in unit of $[\mathrm{mN} / \mathrm{m}]$

\section{REFERENCES}

(1) Ashoori, E.; Marchesin, D.; Rossen, W. R. Roles of Transient and Local Equilibrium Foam Behaviour in Porous Media: Traveling Wave. Colloids Surf., A 2011, 337 (1-3), 228-242.

(2) Apaydin, O. G.; Kovscek, A. R. Surfactant Concentration and End Effects on Foam Flow in Porous Media. Transp. Porous Media 2001, 43, 511-536.

(3) Baghdikian, S.; Handy, L. L. Flow of Foaming Solutions in Porous Media. In Modification of Chemical and Physical Factors in Steam-flood to Increase Heavy Oil Recovery; Yortsos, Y. C., Ed.; U. S. Dept. Energy Ann. Rep., DOE/BC/14126-14: Bartlesville, OK, 1990.

(4) Chou, S. I. Conditions for Generating Foam in Porous Media. In Paper SPE22628, Presented at the SPE Annual Technical Conference and Exhibition, Dallas, TX, 1991.

(5) Douglas, J.; Blair, P. M.; Wagner, R. J. Calculation of linear waterflood behaviour including the effect of capillary pressure. Trans. AIME 1958, 214, 96-102.

(6) Falls, A. H.; Hirasaki, G. J.; Patzek, T. W.; Gauglitz, P. A.; Miller, D. D.; Ratulowski, J. Development of a mechanistic foam simulator: the population balance and generation by snap-off. SPE Reservoir Eng. 1988, 3 (3), 884-892.

(7) Friedmann, F.; Chen, W. H.; Gauglitz, P. A. Experimental and simulation study of high-temperature foam displacement in porous media. SPE Reservoir Eng. 1991, 6 (1), 37-45.

(8) Friedmann, F.; Smith, M. E.; Guice, W. R.; Gump, J. M.; Nelson, D. G. Steam-foam mechanistic field trial in the midway-sunset field. SPE Reservoir Eng. 1994, 9 (4), 297-304.

(9) Gauglitz, P. A.; Radke, C. J. The Dynamic of Liquid Film Breakup in Constricted Cylindrical Capillaries. 1989, J. Colloid Interface Sci. 134, 1.14

(10) Gauglitz, P. A.; Friedmann, F.; Kam, S. I.; Rossen, W. R. Foam Generation in Homogeneous Porous Media. Chem. Eng. Sci. 2002, 57, 4037-4052.

(11) Huh, D. G.; Handy, L. L. Comparison of Steady- and UnsteadyState Flow of Gas and Foaming Solution in Porous Media. SPE Reservoir Eng. 1989, 4, 77.

(12) Jones, S. A.; Laskaris, G.; Vincent-Bonnieu, S.; Farajzadeh, R.; Rossen, W. R. Effect of Surfactant Concentration on Foam: From Coreflood Experiments to Implicit-Texture Foam-Model Parameters. J. Ind. Eng. Chem. 2016, 37, 268-276.

(13) Kam, S. I.; Rossen, W. R. A Model for Foam Generation in Homogeneous Media. SPE J. 2003, 8 (4), 417-42. SPE-87334-PA

(14) Kam, S. I.; Nguyen, Q. P.; Li, Q.; Rossen, W. R. Dynamic Simulations With an Improved Model for Foam Generation, SPE J. 2007, 35-38, DOI: 10.2118/90938-MS

(15) Kam, S. I. Improved Mechanistic Foam Simulation with Foam Catastrophe Theory. Colloids Surf., A 2008, 318 (1-3), 62-77. 
(16) Kovscek, A. R.; Patzek, T. W.; Radke, C. J. A Mechanistic Population Balance Model for Transient and Steady-State Foam Flow in Boise Sandstone. Chem. Eng. Sci. 1995, 50 (23), 3783-3799.

(17) Khatib, Z. R.; Hirasaki, G. J.; Falls, A. H. Effects of Capillary Pressure on Coalescence and Phase Mobilities in Foams Flowing Through Porous Media. SPE Reservoir Eng. 1988, 3 (3), 919-926. SPE-15442-PA.

(18) Kyte, J. R.; Rapoport, L. A. Linear Waterflood Behaviour and End Effects in Water-Wet Porous Media. JPT, J. Pet. Technol. 1958, 213, 47-50.

(19) Lake, L. W.; Johns, R. T.; Rossen, W. R.; Pope, G. A. Fundamentals of Enhanced Oil Recovery; Society of Petroleum Engineers, 2014, ISBN 978-1-61399-407-8.

(20) Ma, K.; Lopez-Salinas, J. L.; Puerto, M. C.; Miller, C. A.; Biswal, S. L.; Hirasaki, G. J. Estimation of Parameters for the Simulation of Foam Flow Through Porous Media. Energy Fuels 2013, 27 (5), 23632375.

(21) Moritis, G. $\mathrm{CO}_{2}$ and $\mathrm{HC}$ Injection Lead EOR Production Increase. Oil Gas J. 1990, 49-81.

(22) Nguyen, Q. P.; Currie, P. K.; Zitha, P. L. J. Determination of Foam Induced Fluid Partitioning in Porous Media Using X-Ray Computed Tomography, Paper SPE80245 presented at the International Symposium on Oilfield Chemistry, Houston, TX, 2003; pp 58.

(23) Perkins, F. M. An Investigation of the Role of Capillary Forces in Laboratory Water Floods. JPT, J. Pet. Technol. 1957, 207, 49-51.

(24) Ransohoff, T. C.; Radke, C. J. Mechanics of Foam Generation in Glass Bead Packs. SPE Reservoir Eng. 1988, 3, 573.

(25) Rossen, W. R.; Gauglitz, P. A. Percolation Theory of Creation and Mobilization of Foams in Porous Media. 1990, AIChE J. 36, 8.1176

(26) Rossen, W. R. Foams in Enhanced Oil Recovery. In Foams: Theory Measurement and Applications; Prud'homme, R. K., Khan, S., Eds.; Marcel Dekker: New York City, 1996.

(27) Simjoo, M.; Zitha, P. L. J. Effects of Oil on Foam Generation and Propagation in Porous Media, Paper SPE 165271 presented at the SPE Enhanced Oil Recovery Conference, Kuala Lumpur, Malaysia, 2013; pp 2-4.

(28) Wonnacott, T. H.; Wonnacott, R. J. Introductory Statistics for Business and Economics; John Wiley and Sons, 1972.

(29) Zhou, Z.; Rossen, W. R. Applying Fractional-Flow Theory to Foam Processes at the "Limiting Capillary Pressure; Society of Petroleum Engineers, 1995, 3, 154, . 